\title{
Energy Dissipation and Noise Correlations in Biochemical Sensing
}

\begin{abstract}
Christopher C. Govern and Pieter Rein ten Wolde
FOM Institute AMOLF, Science Park 104, 1098 XG Amsterdam, Netherlands

(Received 26 June 2014; revised manuscript received 7 October 2014; published 16 December 2014)

To measure chemical concentrations, cells need to extract information from stochastic receptor signals via signaling networks which are also inherently stochastic. Here, we study how the accuracy of sensing depends on the correlations between these extrinsic and intrinsic sources of noise. We find that the sensing precision of signaling networks that are not driven out of equilibrium is fundamentally limited by the fluctuation-dissipation theorem, which generates a tradeoff between the removal of extrinsic and intrinsic noise. As a result, the sensing precision of equilibrium systems is limited by the number of receptors; the downstream network can never improve sensing. To lift the tradeoff, energy dissipation is essential. This allows the receptor to transduce the signal as a catalyst and enables time integration of the receptor state. To beat the sensing limit of equilibrium systems, a canonical nonequilibrium signaling network based on the push-pull motif needs to dissipate at least $1 k_{\mathrm{B}} T$ per receptor.
\end{abstract}

DOI: $10.1103 /$ PhysRevLett.113.258102

Living cells can measure chemical concentrations with extraordinary precision [1-3], but how they do so remains poorly understood [1-16]. Cells measure chemical concentrations via receptors on their surface. These measurements are inevitably corrupted by noise that arises from the stochastic arrival of ligand molecules by diffusion and from the stochastic binding of the ligand to the receptor. Cellular signaling networks have to remove this noise extrinsic to the cell as much as possible. These networks, however, are also stochastic in nature, which means that they will also add noise to the transmitted signal. Most studies on the accuracy of sensing have ignored this intrinsic noise of the signaling network [1-10,1214,16,17]. They essentially assume that it can be made arbitrarily small and that the extrinsic noise in the input can be filtered with arbitrary precision by simply integrating the receptor signal for longer. However, the extrinsic and intrinsic noise are not generally independent [18]. This raises the question how the accuracy of sensing depends on the correlations between them.

Cell use both equilibrium and nonequilibrium motifs to sense their environments (Fig. 1). Protein binding and protein sequestration are equilibrium signaling motifs that are omnipresent in signal transduction pathways; bacterial one-component systems like ROCR provide well-studied examples [19]. Many signaling pathways, including twocomponent systems in bacteria [20] and mitogen-activated protein kinase (MAPK) pathways in eukaryotes [21], are, however, driven out of thermodynamic equilibrium by the turnover of fuel, which leads to the dissipation of heat. Here, we address the question what the sensing limits of equilibrium sensing systems are, and how driving these systems out of equilibrium can improve the precision of sensing. We will show that the answer is intimately
PACS numbers: 87.16.Xa, 05.70.-a, 87.10.Vg, 87.18.Tt

connected to the correlations between extrinsic and intrinsic noise.

We start by studying sensing systems that are not driven out of thermal equilibrium. We show how the sensing precision in these systems is fundamentally bounded by thermodynamic laws. This reveals a fundamental tradeoff between the removal of extrinsic noise in the receptor signal and the suppression of intrinsic noise in the processing network: decreasing one noise source necessarily increases the other. The reason is that in equilibrium systems, the energy of ligand binding is used to change the state of the downstream readout, which inevitably couples receptorligand binding to receptor-readout binding (Fig. 1). As a consequence, equilibrium systems can only reduce the sensing error by increasing the number of receptors. In these systems, adding downstream components can never improve the precision of sensing.

To reduce the extrinsic and intrinsic noise simultaneously, the correlations between them need to be lifted. To this end, energy and the receptor need to be employed (a)

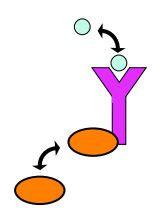

(b)

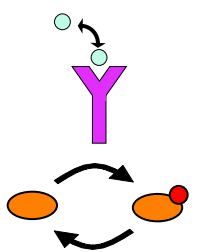

FIG. 1 (color online). Different sensing strategies. (a) In equilibrium systems, the energy of ligand binding is used to change the state of the readout. Here, ligand binding drives readout unbinding: it lowers the system's free energy, overcoming the rise in free energy due to readout unbinding. (b) In nonequilibrium systems, like the Goldbeter-Koshland push-pull motif, fuel turnover drives chemical modification of the readout, and the receptor catalyzes the modification when ligand bound. 
differently (Fig. 1). Rather than using the energy of ligand binding to change the state of the readout, the system should use fuel. This makes it possible to change the state of the readout via chemical modification, with the receptor catalyzing the modification. Receptor-ligand binding and receptor-readout binding are then uncoupled, and the readout molecules provide a stable memory of the history of the receptor state. This yields the mechanism of time integration in which the extrinsic noise in the receptor state and the intrinsic noise of the signaling network can be reduced simultaneously.

We end by addressing the question how much nonequilibrium sensing systems must be driven out of equilibrium to sense more precisely than equilibrium systems. We therefore study a network that combines both modes of signal transmission. We show that dissipation only improves the accuracy of sensing if more than about $1 k_{B} T$ is dissipated per receptor.

Consider a cell with $R_{T}$ receptor molecules on its surface that independently bind ligand, $R+L \rightleftharpoons R L$. Downstream of the receptor is an as yet unspecified network with a readout species $X$. The question is how accurately the cell can infer the ligand concentration $c$ from the instantaneous level $x$ of $X$, by inverting the input-output relation $\bar{x}(c)$. Linearizing $\bar{x}(c)$, and using error propagation, the cell's uncertainty about $c$ is [4]:

$$
\left(\frac{\delta c}{c}\right)_{x}^{2}=\frac{1}{c^{2}} \frac{\sigma_{x}^{2}}{\left(\frac{d \bar{x}}{d c}\right)^{2}}=\frac{\sigma_{x}^{2}}{\left(\frac{d \bar{x}}{d \mu_{L}}\right)^{2}},
$$

where $\mu_{L}=\mu_{0}+\log c$ is the ligand's chemical potential, in units of $k_{\mathrm{B}} T=1$. The uncertainty is low if the average readout level $\bar{x}$ responds sensitively to changes in ligand concentration, as measured by the gain $d \bar{x} / d c$, but $x$ is not noisy, as measured by the variance $\sigma_{x}^{2}$.

If the receptor-ligand complex itself is taken as the readout, then the error is

$$
\left(\frac{\delta c}{c}\right)_{\mathrm{RL}}^{2}=\frac{1}{p(1-p)} \frac{1}{R_{T}} \geq \frac{4}{R_{T}},
$$

since both $\sigma_{R L}^{2}$ and $\left(d \overline{R L} / d \mu_{L}\right)$ equal $R_{T} p(1-p)$, where $p$ is the probability a receptor is bound to ligand [22]. The sensing error is given by the error $1 /[p(1-p)]$ of a single measurement divided by the number of receptors $R_{T}$, because each receptor provides an independent measurement. Clearly, the sensing error is limited by the total number of receptors.

Cells can reduce the error in Eq. (2) with downstream networks that time integrate the receptor state $[1,4-11$, $14,15,17]$. This requires a memory of the past receptor states. This memory can be implemented, e.g., by longlived molecular species [10]. Equilibrium systems can have these, and we might thus expect that they can reduce the sensing error past the bound set by the number of receptors.
Inspired by one component signaling networks [19], we start by considering cytoplasmic readout molecules $x$ that directly bind ligand-free receptors [Fig. 1(a)]: $R+$ $L \rightleftharpoons R L, R+x \underset{k_{\mathrm{r}}}{\stackrel{k_{\mathrm{f}}}{\rightleftharpoons}} R x$. Solving the associated Langevin equations shows that the dynamics of the output $x$ around its mean $\bar{x}$ is given by the time-integrated fluctuations $\delta R L(t)$ in the receptor state plus noise $\eta(t)$ in the downstream network [22]:

$$
\delta x(t)=\int_{-\infty}^{t} d t^{\prime} e^{-\left(t-t^{\prime}\right) / \tau_{I}}\left[\beta \delta R L\left(t^{\prime}\right)+\eta\left(t^{\prime}\right)\right],
$$

where $\beta=k_{\mathrm{f}} \bar{x}$ and $\tau_{I}=1 /\left[k_{\mathrm{f}}(\bar{x}+\bar{R})+k_{\mathrm{r}}\right]$ is the integration time. The latter can be made arbitrarily large by slowing down the readout dynamics, i.e., by lowering $k_{\mathrm{f}}$ and $k_{\mathrm{r}}$. This suggests that even equilibrium networks can completely filter the extrinsic noise in the receptor states. Indeed, we might assume that the intrinsic noise can be suppressed by a suitable choice of the parameters, and that equilibrium systems can thereby reduce the sensing error to zero, just like nonequilibrium sensing systems [11,15]. This, however, is not the case, as we show next.

We can compute the variance $\sigma_{x}^{2}=\langle\delta x\rangle^{2}$, either via the linear-noise approximation [22] or directly from Eq. (3), and plug the result together with the gain $d \bar{x} / d c$ into Eq. (1) to find:

$$
\left(\frac{\delta c}{c}\right)^{2}=\frac{(R+R L)[(R x)(x)+(R+R L)(R x+x)]}{(R L)^{2}(R x)(x)} .
$$

With the sensing error in this form, one can verify that the minimal sensing error is $4 / R_{T}$, thus no better than Eq. (2). Clearly, the error is never lower than the bound set by the number of receptors, regardless of the integration time, the readout copy number, or other network parameters.

This raises the paradox of a network that time integrates the receptor fluctuations yet cannot reduce the sensing error with it. The resolution of the paradox is that in this system the intrinsic and extrinsic noise are correlated. To examine this, we use Eq. (3) to decompose the variance of the output $\sigma_{x}^{2}=\langle\delta x\rangle^{2}$ into the sum of the extrinsic noise $\sigma_{\mathrm{ex}, x}^{2} \equiv$ $\beta^{2} K_{\delta R L, \delta R L}$ and the intrinsic noise $\sigma_{\mathrm{in}, x}^{2} \equiv \beta K_{\delta R L, \eta}+K_{\eta, \eta}$, where $K_{A, B}=\int_{-\infty}^{t} \int_{-\infty}^{t} e^{-\left(t-t_{1}{ }^{\prime}\right) / \tau_{1}} C_{A, B}\left(t_{1}{ }^{\prime}, t_{2}{ }^{\prime}\right) e^{-\left(t-t_{2}{ }^{\prime}\right) / \tau_{1}} d t_{1}$ $d t_{2}$ with the correlation function $C_{A B}\left(t_{1}, t_{2}\right)=\left\langle A\left(t_{1}\right)\right.$ $\left.B\left(t_{2}\right)\right\rangle$. This decomposition is not unique, because we could have assigned the coupling term $\beta K_{\delta R L, \eta}$ to the extrinsic noise term instead of to the intrinsic noise one. In the form above, however, the extrinsic noise term features a canonical temporal average of the input (receptor) fluctuations $[18,28,29]$. In this form, the extrinsic noise term can be made arbitrarily small by increasing the integration time $\tau_{I}$ [see Fig. S(1)]. Importantly, however, we find that when decreasing the extrinsic noise $\sigma_{\mathrm{ex}, x}^{2}$ would reduce the sensing error $(\delta c / c)^{2}$ [given by 
$\sigma_{x}^{2}=\sigma_{\mathrm{ex}, x}^{2}+\sigma_{\mathrm{in}, x}^{2}$ divided by the gain squared, see Eq. (1)] below $4 / R_{T}$, then the intrinsic noise $\sigma_{\mathrm{in}, x}^{2}$ increases by at least the same amount [see Fig. S(2)]. No matter which parameter is varied, and no matter how the total noise $\sigma_{x}^{2}$ is decomposed into an extrinsic noise term and an intrinsic noise one, the sensing error never falls below $4 / R_{T}$. The physical origin is that in equilibrium systems receptorligand binding and receptor-readout binding are coupled. As we elucidate below and in [22], in these systems, a change in the state of the readout necessarily affects the input $R L(t)$. As a result, the intrinsic fluctuations in the activation and deactivation of the readout, modeled by $\eta(t)$, become correlated with the extrinsic fluctuations in the input $R L(t): K_{\delta R L, \eta} \neq 0$. It is this cross-correlation, originating from the coupling between receptor-readout binding and receptor-ligand binding, that leads to a fundamental tradeoff between the removal of extrinsic and intrinsic noise in equilibrium systems.

Signaling networks are usually far more complicated than a single readout molecule that binds the receptor, and it has been shown that additional network layers can reduce the sensing error [10]. This raises the question whether a more complicated equilibrium network can overcome the limit set by the number of receptors. Searching over all possible network topologies to systematically address this question is difficult, if not impossible. However, equilibrium systems are fundamentally bounded by the laws of equilibrium thermodynamics, regardless of their topology. One such law is the fluctuation-dissipation theorem.

Specifically, for any readout $x$ in an equilibrium system, the fluctuation-dissipation theorem states that the gain $d \bar{x} / d \mu_{L}$ is equal to the covariance $\sigma_{x, R L}^{2}$ of the fluctuations in the readout and the ligand-bound receptor $R L$, since $R L$ (or a complex containing it) is the species conjugate to $\mu_{L}$ [30]. Then, for any readout $x$, [Eq. (1)]:

$$
\left(\frac{\delta c}{c}\right)_{x}^{2}=\frac{\sigma_{x}^{2}}{\left(d \bar{x} / d \mu_{L}\right)^{2}}=\frac{\sigma_{x}^{2}}{\left(\sigma_{x, R L}^{2}\right)^{2}} .
$$

To see that this is always bounded by the number of receptors, note that if the receptors themselves are taken as the readout, the sensing error is $(\delta c / c)_{R L}^{2}=1 / \sigma_{R L}^{2}$. By combining this expression with the expression for $(\delta c / c)_{x}^{2}$, it follows that no readout is better for sensing than the receptors:

$$
\left(\frac{\delta c}{c}\right)_{x}^{2}=\frac{\sigma_{x}^{2} \sigma_{R L}^{2}}{\left(\sigma_{x, R L}^{2}\right)^{2}}\left(\frac{\delta c}{c}\right)_{R L}^{2} \geq\left(\frac{\delta c}{c}\right)_{R L}^{2},
$$

since $\left|\sigma_{x, R L}^{2}\right| / \sqrt{\sigma_{x}^{2} \sigma_{R L}^{2}}$ is a correlation coefficient, and so is always less than 1 in magnitude. A downstream signaling network can never improve the accuracy of sensing.

This relation leads to quantitative bounds on the sensing capacity of equilibrium networks. In general, the variance
$\sigma_{R L}^{2}$, and hence $(\delta c / c)_{R L}^{2}$, depends on the particular network, since downstream molecules can affect $\sigma_{R L}^{2}$ by binding the receptor. However, the variance of any random variable $0 \leq Y \leq a$ is $\sigma_{Y}^{2} \leq a^{2} / 4$. Thus, for any network, $\sigma_{R L}^{2} \leq R_{T}^{2} / 4$ since $0 \leq R L \leq R_{T}$. Then, for equilibrium systems, the fundamental lower bound on the fractional error in the concentration estimate is:

$$
\left(\frac{\delta c}{c}\right)_{x}^{2} \geq \frac{4}{R_{T}^{2}}
$$

This proves that in equilibrium systems, the sensing precision is fundamentally limited by the number of receptors.

Interestingly, for all equilibrium networks in which the receptors bind the ligand noncooperatively, $\sigma_{R L}^{2} \leq R_{T}$ [22]. Hence, for all noncooperative equilibrium networks, including the special case of Eq. (2), we have

$$
\left(\frac{\delta c}{c}\right)_{x}^{2} \geq \frac{1}{R_{T}}
$$

This bound for noncooperative equilibrium networks is typically (i.e., when $R_{T} \geq 4$ ) worse than the fundamental bound for all equilibrium networks, given by Eq. (7). This shows that cooperative ligand binding is necessary for reaching the latter bound. In [22], we show that the cooperative binding of ligand to receptor clusters makes it indeed possible to beat the noncooperative bound of Eq. (8) in equilibrium systems. Whether these cooperative systems can actually reach the fundamental bound for all equilibrium sensing systems [Eq. (7)] remains, however, an open question [22]. Lastly, our observation that cooperative binding can lower the bound of equilibrium systems is in contrast to the observation of Skoge et al. that cooperative interactions between neighboring receptors cannot improve sensing in nonequilibrium sensing systems [13] (see [22] for a detailed discussion).

The different species in a network can also be viewed as nodes through which information about the ligand flows. The data processing inequality [31] guarantees for any network that no readout can have more information about the ligand concentration encoded in its time trace than the receptor has in its time trace: $I\left(x_{[0, T]}(t) ; \mu_{L}\right) \leq$ $I\left(R L_{[0, T]}(t) ; \mu_{L}\right)$, where $I$ is the mutual information between the arguments and $x_{[0, T]}(t)$ indicates the time trace of $x$ from time 0 to time $T$. For equilibrium networks, we can show that the data processing inequality guarantees a stronger result [22]: no readout has more information about the ligand than the receptors at any given time: $I\left(x(T) ; \mu_{L}\right) \leq I\left(R L(T) ; \mu_{L}\right) \leq \log _{2}\left(R_{\mathrm{T}}+1\right)$, and therefore, the information in the instantaneous level of the readout is bounded by the number of receptors. This statement is the information-theoretic analogue of Eqs. (6) and (7). The history of receptor states does contain more 
information about the ligand concentration than the instantaneous receptor state, but our results show that an equilibrium signaling network cannot exploit this: its output contains only as much information as the instantaneous receptor state; it does not encode the history of receptor states in any informative way, whether by time integration or any other method.

The tradeoffs faced by equilibrium networks are manifestations of their time reversibility [32]. Time-reversible systems have symmetry between their past and future: if they integrate the past [as in Eq. (3)], they must also integrate and hence, perturb the future; here, readout binding to the receptor will affect future ligand binding. Concomitantly, in a time reversible system, there is no sense of "upstream" and "downstream", concepts which rely on a direction of time [32]. Although we have referred to the molecule $x$ as a "readout" of the ligand concentration, the ligand is just as much a readout of $x$. In an equilibrium system, the sensing error, like any static quantity, can only depend on ratios of time scales (i.e., the equilibrium constants), which is another way of seeing that increasing the "integration time" cannot improve sensing.

While equilibrium systems can only increase the number of concentration measurements by increasing the number of receptors, nonequilibrium systems can also raise the number of measurements by increasing the number of measurements per receptor. These different restrictions ultimately arise from how the two systems employ receptors and energy for signaling.

Equilibrium systems sense by harvesting the energy of ligand binding, capitalizing on the chemical work that is done in the environment to change the ligand concentration. This energy is used to propagate the signal through the downstream network; in the simple system studied here, for example, the energy of ligand binding is used to drive the readout molecule from the receptor. However, detailed balance then dictates that the receptor-readout binding also influences receptor-ligand binding, thus perturbing the signal and creating correlations between intrinsic and extrinsic noise.

Energy dissipation - fuel turnover - is needed to break the tradeoff between extrinsic and intrinsic noise. While in equilibrium systems, the stability of the downstream signaling proteins relies on physical interactions with the receptor molecules, in nonequilibrium systems, the energy to change their state is provided by the chemical fuel [Fig. 1(b)]. The receptor catalyzes the chemical modification of the readout, but after modification, the receptor and readout become decoupled, and each readout molecule provides a stable memory of the receptor state when it was modified. It is this feature that allows these nonequilibrium systems to time integrate the receptor state without increasing the intrinsic noise, and to increase the number of concentration measurements per receptor. Indeed, in these systems, the sensing error is no longer limited by the number of receptors $[11,15]$.
However, nonequilibrium sensing not only requires time to integrate the receptor state [1], but also downstream readout molecules to store the history of the receptor states [15], and energy to store these states reliably [11,15]. This raises the question which sensing strategy can sense more accurately, given these cellular resources. In [15], we show that the sensing error of the canonical, nonequilibrium motif of Fig. 1(b) is bounded by

$$
\left(\frac{\delta c}{c}\right)^{2} \geq \operatorname{MAX}\left(\frac{4}{R_{T}\left(1+\tau_{r} / \tau_{c}\right)}, \frac{4}{X_{T}}, \frac{4}{w}\right),
$$

where $X_{T}$ is the number of readout molecules; $\tau_{c}$ is the correlation time of the receptor signal; $\tau_{r}$ is the relaxation time of the network, which sets the integration time; and $w$ is the energy dissipated during the integration time. Comparing the above expression with that for equilibrium systems without cooperative binding [Eq. (8)] suggests that this nonequilibrium system can sense more accurately than equilibrium systems only when there is at least one readout molecule per receptor, and when the amount of energy dissipated during the integration time is at least $1 k_{B} T$ per receptor.

To test this, we have considered a network that combines both sensing strategies (Fig. 2). Numerically optimizing the sensing error over all parameters shows that when the energy per receptor, $w / R_{T}$, is less than a few $k_{B} T$, the optimized system employs parameters that correspond to the equilibrium strategy of sequestration, while if $w / R_{T}$ is higher, it uses

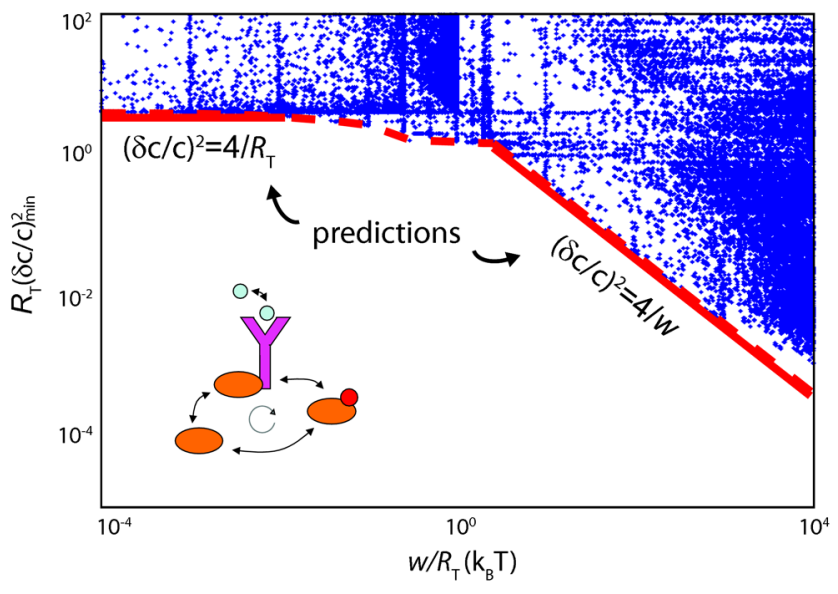

FIG. 2 (color online). The different resource requirements for equilibrium and nonequilibrium sensing lead to a tradeoff between these two modes. The tradeoff is illustrated for a network that combines both modes: $R L+x \rightleftharpoons R L x, R L x \rightleftharpoons$ $R L+x^{*}, x^{*} \rightleftharpoons x$. The blue dots show the sensing error for different parameter values [22]. The solid red lines show, respectively, the predictions of Eqs. (2) and (9), while the dashed red line shows the minimum sensing error obtained from the numerical optimization. When the energy per receptor $w / R_{T}$ is less than a few $k_{B} T$, the optimized system employs the equilibrium strategy, with bound $4 / R_{T}$, while if it is higher, it uses the nonequilibrium strategy, achieving the bound $4 / w$. 
parameters corresponding to the nonequilibrium strategy of catalysis to transmit the signal (Fig. 2), in accordance with the prediction based on Eqs. (2) and (9).

One-and two-component signaling networks provide a case study for the tradeoff between equilibrium and nonequilibrium sensing. One-component systems consist of adaptor proteins which can bind an upstream ligand and a downstream effector, while two-component systems are similar to the push-pull network studied here, consisting of a kinase (receptor) and its substrate. Interestingly, some adaptor proteins, like RocR, contain the same ligand-binding domain as the kinase and the same effector-binding domain as the substrate of a two-component system, i.e., NtrB-NtrC [19]. It has been suggested that one-component systems have evolved into two-component systems to facilitate transfer of signals from the membrane to the nucleus [19]. However, our analysis reveals that equilibrium networks can also transmit signals across space. Indeed, our results suggest that equilibrium and nonequilibrium sensing motifs are alternative signaling strategies, selected because of different resource selection pressures. It is tempting to speculate that when sensing precision is critical, but space for receptors on the membrane is limiting, nonequilibrium sensing becomes essential, because it makes it possible to take more concentration measurements per receptor.

We thank Jeroen van Zon for a critical reading of the Letter. This work is part of the research program of the Foundation for Fundamental Research on Matter (FOM), which is part of the Netherlands Organization for Scientific Research (NWO).

[1] H. C. Berg and E. M. Purcell, Biophys. J. 20, 193 (1977).

[2] V. Sourjik and H. C. Berg, Proc. Natl. Acad. Sci. U.S.A. 99, 123 (2002).

[3] M. Ueda and T. Shibata, Biophys. J. 93, 11 (2007).

[4] W. Bialek and S. Setayeshgar, Proc. Natl. Acad. Sci. U.S.A. 102, 10040 (2005).

[5] K. Wang, W.-J. Rappel, R. Kerr, and H. Levine, Phys. Rev. E 75, 061905 (2007).

[6] W.-J. Rappel and H. Levine, Phys. Rev. Lett. 100, 228101 (2008).

[7] R. G. Endres and N. S. Wingreen, Phys. Rev. Lett. 103, 158101 (2009).

[8] B. Hu, W. Chen, W.-J. Rappel, and H. Levine, Phys. Rev. Lett. 105, 048104 (2010).
[9] T. Mora and N. S. Wingreen, Phys. Rev. Lett. 104, 248101 (2010).

[10] C. C. Govern and P. R. ten Wolde, Phys. Rev. Lett. 109, 218103 (2012).

[11] P. Mehta and D. J. Schwab, Proc. Natl. Acad. Sci. U.S.A. 109, 17978 (2012).

[12] M. Skoge, Y. Meir, and N. S. Wingreen, Phys. Rev. Lett. 107, 178101 (2011).

[13] M. Skoge, S. Naqvi, Y. Meir, and N. S. Wingreen, Phys. Rev. Lett. 110, 248102 (2013).

[14] K. Kaizu, Wiet de Ronde, J. Paijmans, K. Takahashi, F. Tostevin, and P. R. ten Wolde, Biophys. J. 106, 976 (2014).

[15] C. C. Govern and P. R. ten Wolde, Proc. Natl. Acad. Sci. U.S.A. 111, 17486 (2014).

[16] A. H. Lang, C. K. Fisher, T. Mora, and P. Mehta, Phys. Rev. Lett. 113, 148103 (2014).

[17] G. Aquino and R. G. Endres, Phys. Rev. E 81, 021909 (2010).

[18] S. Tănase-Nicola, P. B. Warren, and P. R. ten Wolde, Phys. Rev. Lett. 97, 068102 (2006).

[19] L. E. Ulrich, E. V. Koonin, and I. B. Zhulin, Trends Microbiol. 13, 52 (2005).

[20] A. M. Stock, V. L. Robinson, and P. N. Goudreau, Annu. Rev. Biochem. 69, 183 (2000).

[21] L. Chang and M. Karin, Nature (London) 410, 37 (2001).

[22] See Supplemental Material at http://link.aps.org/ supplemental/10.1103/PhysRevLett.113.258102, which includes Refs. [23-27].

[23] C. W. Gardiner, Handbook of Stochastic Methods (Springer, Berlin, 1985).

[24] E. Ziv, I. Nemenman, and C. H. Wiggins, PLoS One 2, e1 077 (2007).

[25] P. A. Samuelson, Foundations of Economic Analysis (Harvard University Press, Cambridge, 1947).

[26] T. Schmiedl and U. Seifert, J. Chem. Phys. 126, 044101 (2007).

[27] T. Hill, Free Energy Transduction in Biology: The Steadystate Kinetic and Thermodynamic Formalism (Academic Press, New York, 1977).

[28] J. Paulsson, Nature (London) 427, 415 (2004).

[29] T. Shibata and K. Fujimoto, Proc. Natl. Acad. Sci. U.S.A. 102, 331 (2005).

[30] H. Risken, Fokker-Planck Equation (Springer, Berlin, 1984).

[31] T. M. Cover and J. A. Thomas, Elements of Information Theory (John Wiley and Sons, Hoboken, 2012).

[32] E. H. Feng and G. E. Crooks, Phys. Rev. Lett. 101, 090602 (2008). 